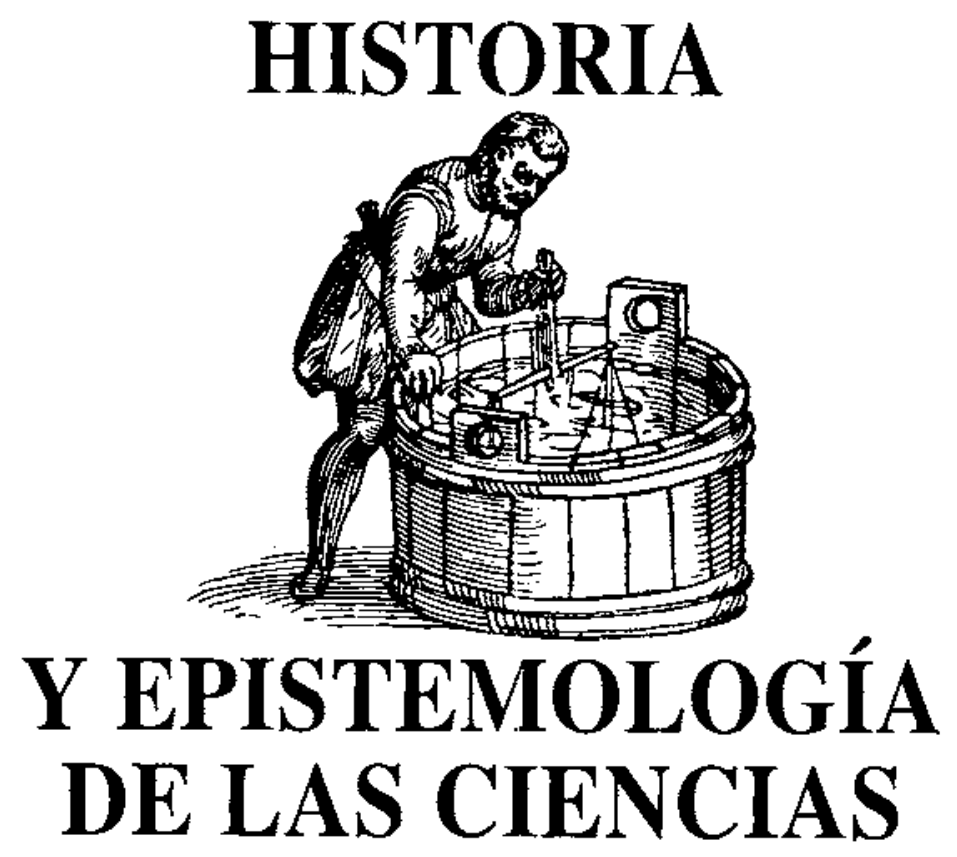

\title{
APLICACIÓN DIDÁCTICA DE LA BALANZA «PESAORO» DE ARQUÍMEDES
}

SALVAT AITÉS, A. ${ }^{1}$ y SÁNCHEZ REAL, J. ${ }^{2}$

'Área de Didáctica de las Ciencias Experimentales. Universidad «Rovira i Virgili». Tarragona.

${ }^{2}$ Escuela Universitaria de Magisterio. Valencia.

\section{SUMMARY}

In this article two methods are exposed -with its practical application in the school-in order to discover whether the golden crown of King Hieron III from Siracusa (III c. bC) was totally made of gold or, on the contrary, the goldsmith who had made it, changed by silver part of the gold given by the King.

\section{INTRODUCCIÓN}

Arquímedes, según la tradición, contribuyó de forma efectiva a todas las especialídades mecánicas helenísticas: construcción de maquinaria bélica y de fortificación militar, construcción de artefactos y obras de utilidad pública -como sistemas de poleas compuestas-, cons- trucción de mecanismos e ingentos espectaculares, diseno de planetarios y esferas celestes y el estudio de centros de gravedad y condiciones de equilibrio de los cuerpos. Tampoco debe olvidarse que la posición de Arquímedes en la corte siracusana apuntaba hacia una 
asociación entre la investigación científica y la ingeniería civil y militar. Asi pues, no se necesita mucho ingenio para ver en Arquímedes el prototipo, no sólo del científico, sino del ingeniero de la época. La anécdota de la corona de oro del rey Hierón de Siracusa es una prueba más de la capacidad de Arquímedes en el campo de la construcción de aparatos de cierta utilidad.

Por otra parte, en una comunicación científica (Salvat 1987) ya se daba cuenta de la deformación introducida por los textos escolares al presentar el mal denominado principio de Arquímedes como consecuencia de dar solución al enigma de la corona de oro del rey Hierón. A la misma conclusión llegó un estudioso (Vega 1989) del sabio griego cuando afirma que «la corona del rey Hierón tiene la misma relación con su tratado de hidrostática como la que tiene la manzana de Woolsthorpe con la gravitación newtoniana».

\section{LA LEYENDA DE LA CORONA, SEGÚN VITRUVIO}

De acuerdo con la narración, el rey de Siracusa, en muestra de agradecimiento a los dioses por los favores recibidos, decidió consagrarles una corona de oro. Al recibir el trabajo del orfebre y pensando que éste podría haberle engañado, sustrayendo una parte del oro entregado y reponiéndola en plata, le encargó a Arquímedos que resolviese el enigma. Según el relato de Vitruvio:

«...(Archîmedes) fue casualmente á bañarse: y al entrar en el solio, observó, que quanto su cuerpo iba ocupando de sitio, tanta agua se derramaba del solio. Inferida de aqui fa resolución de su encargo, saltó luego del solio lleno de alegria, y partiendo desnudo hácia su casa, iba repitiendo en alta voz haber hallado lo que buscaba: pues corriendo clamaba continuamente en Griego eyreca, eyreca (lo hallé, lo hallé). Sobre este principio, se dice que previno una porción de oro y otra de plata, cada una de tgual peso al de ta corona. Luego llenó de agua hasta el borde un vaso capaz, en el qual metió la porcion de plata, la qual expelió de! vaso tanta agua quanto era su volumen. Sacó la platá, y volviendo á llenar el vaso de agua como estaba antes hasta el borde, cupo un sextario. Con esto tuvo averiguado quanta agua correspondia á una determinada porción de plata. Sabido esto, metió tambien la porción de oro en el vaso lleno, y sacada, añadió como antes una medida de agua, y advirtió que no habia salido tanta como la primera vez, sino tanta menos quanto menos voluminosa era una masa de oro que una de plata de pesos iguales. Finalmente, vuelto á llenar el vaso, y metida en el agua la corona misma, halló haber expelido esta mas agua que la masa de oro igual en peso á ella; y por esta mayor expulsión de agua en la corona arguyó la mezcla de plata en el oro, y el hurto manifiesto del artífice» (Ortiz 1787).

Del relato se concluye que Arquímedes determinó si la corona llevaba una mezcla de plata de forma relativamente sencilla. Para ello tomó dos trozos metálicos de ta misma masa que la corona, uno de plata y el otro de oro, y por desplazamiento del agua calculó sus respectivos volúmenes. Despućs, por el mismo método, fijó el volumen de lat corona; si este volumen se encontraba entre el de la pieza de oro puro y el de la plata pura, el oro cstaba ligado. Si coincidía con el de la pieza de oro puro, la corona no estaba aleada. En tanto en cuanto más se aproximase el volumen de la coronáa al de la pieza de plata pura, mayor serfa el hurto del joyero. Así, usando una propiedad característica de los metales (la densidad, en lenguaje moderno) pudo resolver el regio problema.

\section{Cálculo de la ley de la corona por el método del desplazamiento en agua}

De las obras de Arquímedes conocidas en la actualidad, unas de tipo matemático destinadas a la demostración de proposiciones sobre áreas de volúmenes de figuras limitadas por líneas o superficies curvas (Sobre la cuadratura de la parábola, Sobre la esfera y el cilindro, ctc.) y otras de tipo estático e hidrostático, en las que los problemas físicos planteados se resuelven por métodos geométricos (Sobre los cuerpos flotantes), se concluye que Arquímedes dominaba perfectamente la teoría y la práctica de las fracciones o razones, ya que en todas las demostraciones, incluso en las de tipo hidrostático, empleaba dicho método (Gamow 1983). Así, argumentando al estilo de Arquímedes, pero en lenguaje actual, el famoso siracusano podría haber deducido, fácilmente, no sólo el hurto del platero, sino, además, la cantidad de oro sustraída.

En el texto de Vitruvio se dice que la porción de plata emplcada (del mismo peso que la corona) «expelió un sextario de aguan. Como el sextario (romano) equivalía a la sexta parte del congio $(3,283$ litros), el volumen de la plata era de $547 \mathrm{~cm}^{3} \mathrm{y}$, cn consecuencia, tenía una masa de $5,475 \mathrm{~kg}$. Por tanto, la masá de la corona también era de $5,745 \mathrm{~kg}$. Ortiz (1787) da un valor de quince libras y media para la corona, que equivalen a $5,075 \mathrm{~kg}$ (a la libra romana se le asigna un valor aproximado de 327,45 gramos).

Tomando como masa de la corona el valor calculado y suponiendo que el orfebre había introducido una cierta cantidad de plata en la corona - cantidad que sólo él conocía-, Arquímedes hubiese podido argumentar en los siguientes términos:

Tomamos dos bloques de oro y de plata, de igual masa que la corona $(5,745 \mathrm{~kg})$ y, por desplazamiento del agua, determinemos el volumen de los tres cuerpos. Supongamos que los resultados obtenidos son los de la figura 1.

Si la corona se hubiese realizado totalmente en oro su volumen sería de $298 \mathrm{~cm}^{3}$, mientras que si el orfebre la hubiese hecho toda de plata el volumen ascendería hasta los $547 \mathrm{~cm}^{3}$. La proporción de plata en la corona vendrá fijada por la relación:

$$
\frac{V-V_{A U}}{V_{A \mathcal{E}}-V_{A U}}
$$


Figura 1

Cálculo de la ley de la corona por el método del desplazamiento en agua.
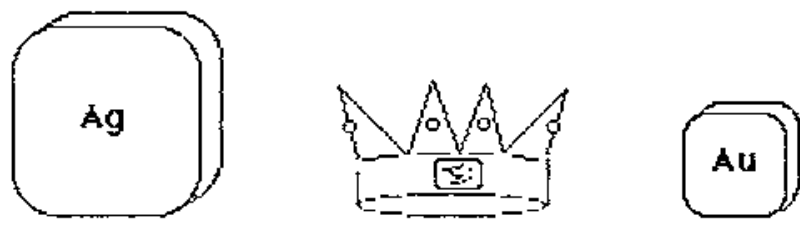

\section{$5,745 \mathrm{~kg}$}

$547 \mathrm{~cm}^{3}$
(200 g) y, por el mismo método, se hallan sus respectivos volúmenes: $74 \mathrm{~cm}^{3}$ para el aluminio y $17 \mathrm{~cm}^{3}$ para el plomo.

La proporción de aluminio en la «corona» será: (30-I7) $/(74-17)=13 / 57=0,228(=23 \%)$, mientras que la de oro valdrá $0,772(=77,2 \%)$.

Desmontando la «corona» en sus partes y pesando, por separado, con una balanza, el aluminio y el plomo se averiguará, de nuevo, su composición: $40 \mathrm{~g}$ de aluminio (20\%) y $160 \mathrm{~g}$ de plomo $(80 \%)$.

Foto 1 en Ia que $V$ simboliza el volumen de la corona; $V_{A E}$, el de Ia pieza de plata, y $V_{A u}$, el del oro. $\left(V-V_{A u}\right)$ representa la diferencia de volumen entre la corona y la masa equivalente de oro puro. Cuando la corona no esté aleada dicha diferencia será nula, mientras que, si fuese enteramente de plata, el cociente $\left(V-V_{A v}\right) /\left(V_{A B}-V_{A v}\right)$ valdria la unidad.

Para el caso de la figura 1, la proporción (en peso) de plata de la corona será (347-298)/(544-298), o lo que es aproximadamente to mismo: $1 / 5$. Es decir, la corona contenía 1 parte de plata por cada 4 partes de oro $(20 \%$ de plata y $80 \%$ de oro, en lenguaje actual), con lo que se deduce que el orfebre había escamoteado $1,149 \mathrm{~kg}$ de oro.

\section{APIJICACIÓN DIDÁCTICA}

El proceso anterior puede usarse para calcular la composición de una «corona» construida por el profesor. Dado que el oro y la plata son metales preciosos y tienen un precio prohibitivo, se usará aluminio $\left(2.700 \mathrm{~kg} / \mathrm{m}^{3}\right)$ y plomo $\left(11.350 \mathrm{~kg} / \mathrm{m}^{3}\right)$. La relación de sus densidades (1/4, aproximadamente) hace que la diferencia de volumen entre dos bloques metálicos de la misma masa sea aun más acusada que si los metales empleados fuesen el oro $\left(19.300 \mathrm{~kg} / \mathrm{m}^{3}\right)$ y la plata $\left(10.500 \mathrm{~kg} / \mathrm{m}^{3}\right)$, con et consiguiente aumento de la diferencia de volúmenes.

El plomo se obtendrá de una chapa de dicho material de $2 \mathrm{~mm}$ de grosor, cortándola en cuadrados de $2,1 \mathrm{~cm}$ de lado; así cada pieza tendrá una masa próxima a los 10 gramos. El aluminio se obtendrá de un perfil cuadrado hueco de dicho metal de $5 \mathrm{~cm}$ de lado y $2 \mathrm{~mm}$ de grosor. Con una sierra se cortarán piezas de $1 \mathrm{~cm}$ de ancho, ya que así se obtendrán trozos de unos $10 \mathrm{~g}$ de masa. Con los restos que quedan al cortar, también se podrán construir pesas de vator inferior a los 10 gramos.

Se formará una corona «aleando» ambos metales, se mezclarán 4 piezas de aluminio con 16 de plomo $(200 \mathrm{~g}$ de masa total), y por desplazamiento del agua de un recipiente $s c$ determinará su volumen $\left(30 \mathrm{~cm}^{3}\right)$. Sc tomarán 20 piezas de aluminio (200 g) y otras 20 de plomo

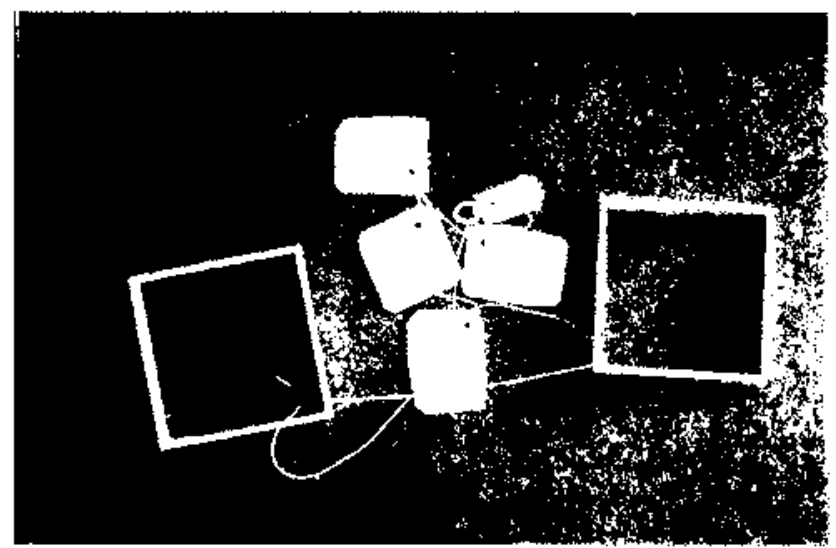

\section{CÁlCULO DE LA LEY DE LA CORONA CON LA BALANZA «PESAORO»}

Después de haber dado solución al problema de la corona de Hierón, Arquímedes construyó una balanza para determinar la cantidad de oro en una aleación de oro y plata. No debe extrañar que una vez resuelto el problema se pusiese a construir, por puro divertimento, un instrumento de estas características, ya que, según Plutarco, Arquímedes «desdeñaba las aplicaciones en el campo de la mecánica y sólo veía en ellas una distracción» (Vega 1986). La descripción de este invento-más propio de nuestra época que no de la de Arquímedesfue incluida en su libro Sobre las balanzas, perdido durante mucho tiempo y que finalmente sc encontró en un manuscrito árabe: Kitab Mizan al-Hikmat, escrito por Abd al-Rahman al-Kahzini y fechado en el año 515 de la hégira mahometana (1095 de nuestra era). En el primer capítulo se dice:

\section{La balanza de Arquímedes y su uso}

«Usamos una batanza muy sensible. Nos proporcionamos dos pesos iguales de plata y de oro y los colocamos en los platillos de una balanza, que se encuentran sumer- 
gidos en el agua. Cuando, a causa del mayor peso específico del oro, la balanza se desequilibre del lado del oro, la volvemos a cquitibrar moviendo el jinete hasta que el brazo de la balanza adopte, de nuevo, la posición horizontal y señalamos la posición que tiene el jinete sobre el brazo (en la parte de la plata). Esta prueba debe ser repetida dos, tres o cuatro veces, en el aire y en el agua. Las distancias del jinete al fulcro de la balanza varían de acuerdo con el peso del jinete.

Figura 2

Balanza «pesaoron de Arquímedes.

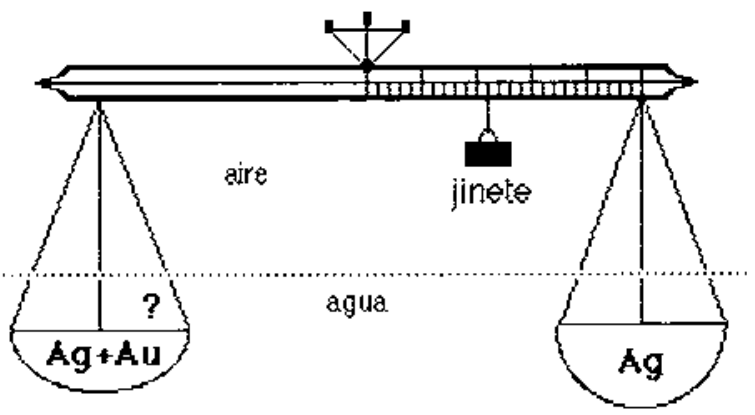

"Pero cuando mezclamos oro con plata y deseamos conocer la proporción de cada uno, debemos proporcionarnos un peso de plata pura igual al peso, en el aire, de la aleación. Después sumergimos los dos platillos en el agua. Ambos platillos deben estar hechos del mismo material y deben hundirse en el agua (como ocurre si son de plata o cobre). Entonces, si la balanza se hunde del lado de la aleación (oro-plata), la equilibramos moviendo el jinete sobre el brazo hasta que éste recupere la posición horizontal. Observamos la posición del jinete y concluimos que este punto de la palanca indica la proporción de oro en la aleación” (Bardis 1984, Pugliese 1988).

Mediante la condición de equilibrio de rotación se demuestra que en una balanza de estas características, cuando se encuentra en la situación descrita en la figura 2, Ia distancia del jinetc al fulcro de la balanza (x) es directamente proporcional a la masa de oro $\left(m_{A u}\right)$ contenida en la aleación,

$\mathrm{x}=\mathrm{g} \cdot \frac{\mathrm{d}_{\mathrm{H} 2 \mathrm{O}}}{\mathrm{m}} \cdot\left[\frac{1}{\mathrm{~d}_{\mathrm{Ag}}} \mathrm{d}_{\mathrm{At}}\right] \cdot \mathrm{m}_{\mathrm{Au}}$

siendo $\mathrm{g}$ la intensidad del campo gravitatorio terrestre; $\mathrm{d}_{\mathrm{H} 2 \mathrm{O}}, \mathrm{d}_{\mathrm{Ag}}, \mathrm{d}_{\mathrm{Av}}$ las densidades del agua, plata y oro, respectivamente; $m$ la masa del jinete, y $\mathrm{m}_{\mathrm{Au}}$, la masa de oro contenida en la aleación. Este resultado significa que, si la corona es completamente de plata $\left(m_{\mathrm{Au}}=0\right)$, el jinete deberá situarse, exactamente, sobre el fulcro, mientras que, si la corona es de oro puro, el jinete cabalgará sobre una posición comprendida entre el fulcro y el extremo del cual pende la masa de plata. La longitud de la escala $y$, por tanto, la sensibilidad del aparato serán directamente proporcionales a la relación $\mathrm{m}_{\mathrm{Au}} / \mathrm{m}$. Esta conclusión tiene su importancia puesto, que al variar la masa del jinete se variará la sensibilidad de la balanza y así se podrá determinar la ley de aleaciones con mucho oro o con poco.

\section{Construcción y calibrado de la balanza}

Se podrá construir una réplica de la balanza de Arquimedes con una varilla de madera de unos $50 \mathrm{~cm}$ de longitud y I cm de diámetro. Se practicará un agujero en la mitad de la varilla, por el que pasará un clavo de $4 \mathrm{~cm}$ de longitud y $2 \mathrm{~mm}$ de diámctro, que jugará el papel de fulcro de la balanza. Para conseguir que la balanza esté equilibrada en el aire, se suspenderá del fulcro y con un cúter se irán sacando pequeñas virutas de madera de la varilla hasta que quede perfectamente nivelada en el aire. En los extremos de la misma se pondrán dos pequeños clavos, en forma de «L», que servirún para colgar los pesos, gracias a dos pequeños hilos de coser.

Foto 2

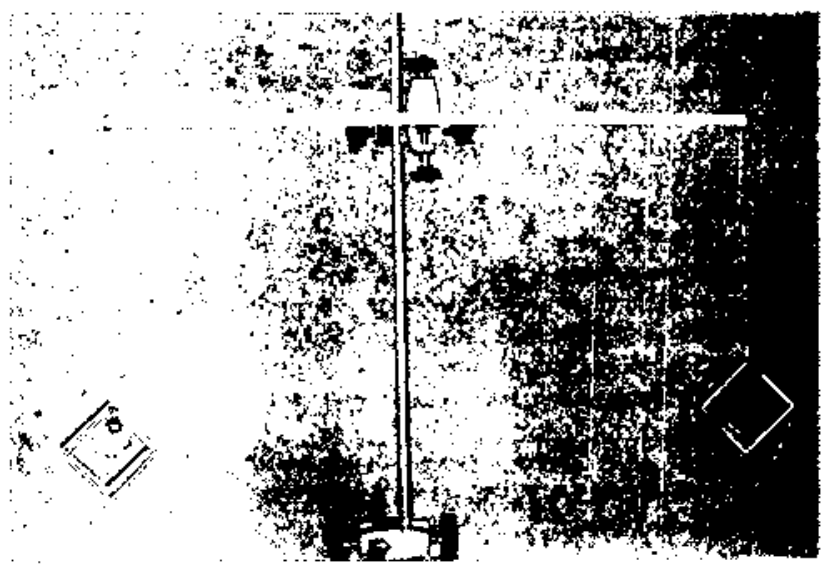

Supongamos que se tienen 104 g de plomo (Pb) en cl platillo izquierdo de la balanza y otros $104 \mathrm{~g}$ de aluminio (Al) en el derecho. La balanza en el aire estará nivelada, mientras que, cuando los platillos se sumergen en el agua (Fig. 3a), la balanza se hunde del lado del plomo y es necesario situar el jinete (de masa $m$ ) a una distancia $s$ del fulcro para nivelarla. En la situación anteriormente descrita, la únjca fuerza que desequilibra la balanza es la diferencia de empuje entre la masa de aluminio y la de plomo (29,3 pondios), que actúa en el brazo de la izquierda. Para volver a nivelarla se usa el jinete.

La ley de la palanca obliga a que el momento originado en el brazo izquierdo equivalga al del Iado derecho (Fig. 3b):

$$
24 \times 29,3=\mathrm{s} \times \mathrm{mg}
$$


Figura 3

Calibrado de la balanza de Arquímedes.

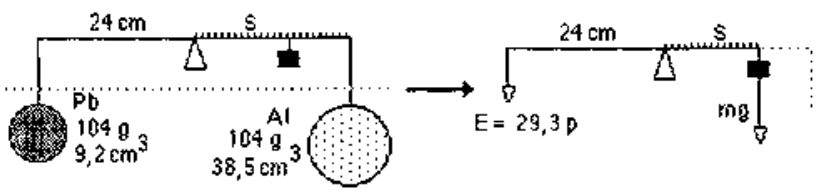

(a)

(b)

El brazo del jinete (s) da la sensibilidad de la balanza en función del peso (mg) del jinete. Jugando con las dos variables se pueden tener distintas sensibilidades para una misma balanza.

Arquímedes no determina la sensibilidad de la balanza de esta forma, sino que lo hace por tanteo, puesto que, una vez, se han equilibrado dos masas iguales de plata y oro, en cl manuscrito se dice: «Esta prueba debe ser repetida dos, tres o cuatro yeces, en el aire y en el agua. Las distancias del jinete al fulcro de la balanza varían de acuerdo con el peso del jinete.»

En nuestro caso, si se quiere que el $100 \%$ de plomo corresponda al máximo de la escala $(20 \mathrm{~cm})$, según (2) la masa del jinete deberá ser: $\mathrm{m}=29,3 \times 24 / 20=35,2 \mathrm{~g}$.

\section{RESULTADOS OBTENIDOS}

Los resultados obtenidos con una «corona» de $104 \mathrm{~g}$ de masa y dos piezas, una de plomo y la otra de aluminio, de la misma masa que la corona, son los que se muestran en la tabla I.

Foto 3

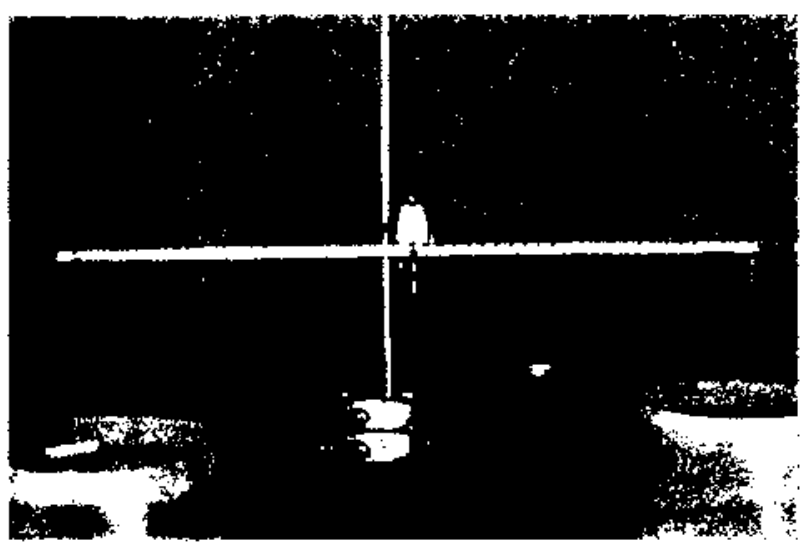

La primera scrie de valores, separada del resto mediante una línea discontinua, corresponde al calibrado de la balanta.
Tabia I

Resultados obtenidos con la balanza de Arquímedes.

\begin{tabular}{|c|c|c|c|c|c|c|}
\hline \multicolumn{3}{|c|}{$<-$ CORONA $\rightarrow$} & $\begin{array}{l}\text { Posicion } \\
\text { jinete }\end{array}$ & $\begin{array}{c}\% \mathrm{~Pb} \\
\text { calcubado }\end{array}$ & $\begin{array}{c}\% \mathrm{~Pb} \\
\text { real }\end{array}$ & $\begin{array}{l}\text { \% error } \\
\text { relativo }\end{array}$ \\
\hline 104,0 & 104,0 & 0,0 & 20,0 & - & - &. \\
\hline 104,0 & 0,0 & 104,0 & 0,0 & 0,0 & 0,0 & 0,0 \\
\hline 104,0 & 20,0 & 84.0 & 3,8 & 19,0 & 19,2 & $-1,2$ \\
\hline 104,0 & 36,7 & 67.3 & 7,0 & 35,0 & 35,3 & $-0,9$ \\
\hline 104,0 & 53.6 & 50,4 & 10,0 & 50,0 & 51,5 & $-3,0$ \\
\hline 104,0 & 64,0 & 40,0 & 11,8 & 59,0 & 61,6 & $.4,1$ \\
\hline 104,0 & 75.8 & 28,2 & 14,0 & 70,0 & 72,9 & $-4,0$ \\
\hline 104,0 & 86,9 & 17,1 & 16,2 & 81,0 & 83,6 & $-3,1$ \\
\hline
\end{tabular}

En las tres primeras columnas se dan los datos relativos a cada una de las siete coronas construidas: masa total (M) y masa de plomo ( $\mathrm{Pb}$ ) y aluminio (Al). En la cuarta columna se indica la posición del jinete sobre la escala cuando la balanza está equilibrada; en la quinta, el porcentaje de plomo contenido en la corona según la lectura de la balanza; mientras que en la sexta se da la composición de la corona en basc a las masas de los metales empleados para construirlas. Por último, en la séptima columna, se indican lis desviaciones producidas con respecto al valor real.

\section{CONCLUSIONES}

Con la realización de esta experiencia se pone de manifiesto la utilidad de la historia de la ciencia en la didáctica de las ciencias experimentales, desde un punto de vista de elemento motivador del aprendizaje.

Por otro lado, la lectura y análisis de textos históricos puede dar una indicación -muy fiable- del tipo de personaje científico que allí se refleja. En este sentido, Arquímedes se nos presenta como un brillante «ingeniero», capaz de construir artefactos más propios de nuestra éproca que no del siglo in a.C.

Des del punto de vista de los procedimientos de los alumnos se puede reproducir, con cierta facilidad, la balanza de Arquímedes, y así pueden darse cuenta del fundamento físico del artilugio.

Por lo que se refiere al propio instrumento de medida -la balanza-, debe indicarse que con un aparato de gran simplicidad técnica se pueden obtener resultados que normalmente se deberían obtener por procedimientos de análisis químico. 


\section{REFERENCIAS BIBLIOGRÁFICAS}

BARDIS, P.D., 1984. An Ancient Find, The Science Teacher, Vol. $51(7)$, pp. 32-33.

GAMOW, G., 1983. Biografia de la fisica. (Alianza Editorial: Madrid).

ORTIZ, J., 1787. De Architectura, de M. Vitruvio Polión. (Imprenta Real: Madrid).
PUGLIESE, S., 1988. La bilancia «pesa-oro» di Archimede, La Fisica nella Scuola, Vol. XXI, pp. 3-5.

SALVAT, A., 1987. Mitos sobre Arquímedes: el asedio a Siracusa y la corona de oro del rey Hieron, Cuadernos de Física y Quimica, Vol. IX, pp. 141-150.

VEGA, L., 1986. Arquimedes: El método. (Alianza Editorial: Madrid). 